\title{
THE M33 METALLICITY PROJECT: RESOLVING THE ABUNDANCE GRADIENT DISCREPANCIES IN M33 ${ }^{1}$
}

\author{
ERIK RosolowsKY \\ Harvard-Smithsonian Center for Astrophysics, 60 Garden Street, MS-66, \\ Cambridge, MA 02138; erosolow@cfa.harvard.edu \\ AND \\ Joshua D. SimON \\ Department of Astronomy, California Institute of Technology, 1200 East California Boulevard, \\ MS 105-24, Pasadena, CA 91125; jsimon@astro.caltech.edu \\ Received 2007 April 12; accepted 2007 November 27
}

\begin{abstract}
We present a new determination of the metallicity gradient in M33, based on Keck LRIS measurements of oxygen abundances using the temperature-sensitive emission line [O III] $\lambda 4363$ in $61 \mathrm{H}$ II regions. These data approximately triple the sample of direct oxygen abundances in M33. We find a central abundance of $12+\log (\mathrm{O} / \mathrm{H})=8.36 \pm$ 0.04 and a slope of $-0.027 \pm 0.012 \mathrm{dex} \mathrm{kpc}^{-1}$, in agreement with infrared measurements of the neon abundance gradient but much shallower than most previous oxygen gradient measurements. There is substantial intrinsic scatter of 0.11 dex in the metallicity at any given radius in M33, which imposes a fundamental limit on the accuracy of gradient measurements that rely on small samples of objects. We also show that the ionization state of neon does not follow the ionization state of oxygen as is commonly assumed, suggesting that neon abundance measurements from optical emission lines require careful treatment of the ionization corrections.
\end{abstract}

Subject headings: galaxies: abundances — galaxies: individual (M33) — galaxies: spiral — H II regions — ISM: abundances - ISM: evolution

Online material: machine-readable table

\section{INTRODUCTION}

M33 was one of the first galaxies in which a radial abundance gradient was recognized (Searle 1971). Beginning with Smith (1975), a series of studies showed that H II region abundances in M33 decline by approximately an order of magnitude over the visible extent of the galaxy (Kwitter \& Aller 1981; Vílchez et al. 1988; Garnett et al. 1992), with a simple exponential providing an accurate fit to the gradient. A typical value for the gradient from this work is $-0.12 \pm 0.02$ dex kpc ${ }^{-1}$ (Vílchez et al. 1988, hereafter V88). More recently, Crockett et al. (2006, hereafter C06) presented new metallicity measurements for a small sample of $\mathrm{H}$ II regions that indicated nearly constant oxygen abundances across M33, with a best-fit gradient of only $-0.012 \pm$ $0.011 \mathrm{dex} \mathrm{kpc}^{-1}$. If confirmed, this very shallow gradient would call into question a variety of results concerning, e.g., the metallicity dependence of the Cepheid period-luminosity relation (Lee et al. 2002) and the $\mathrm{CO}-\mathrm{H}_{2}$ conversion factor (Rosolowsky et al. 2003 ) that depend on the abundance gradient in M33. To complicate matters further, infrared measurements of the neon abundance gradient, which should be less sensitive to systematic effects such as extinction and the temperature of the $\mathrm{H}$ in region, yield an intermediate gradient of $-0.034 \pm 0.015 \mathrm{dex} \mathrm{kpc}^{-1}$ that is not in very good agreement with any of the published oxygen gradients from optical data (Willner \& Nelson-Patel 2002, hereafter WNP02).

\footnotetext{
1 Data presented herein were obtained at the W. M. Keck Observatory, which is operated as a scientific partnership among the California Institute of Technology, the University of California, and the National Aeronautics and Space Administration. The Observatory was made possible by the generous financial support of the W. M. Keck Foundation.
}

In this paper we present new, direct metallicity measurements based on detections of the temperature-sensitive [O III] $\lambda 4363$ line in $61 \mathrm{H}$ II regions in M33, approximately tripling the sample available in the literature for M33 (or any other galaxy). We use these data to provide an improved measurement of the abundance gradient in M33. In $\S 2$ we describe the observations, data reduction, and derivation of metallicities. In $\S 3$ we determine the oxygen abundance gradient in M33, attempt to derive neon abundances, and discuss some of the implications of our results. We briefly summarize the paper in $\S 4$.

\section{OBSERVATIONS AND DATA REDUCTION}

\subsection{Observations}

The M33 Metallicity Project is a long-term program in which we plan to obtain deep spectroscopy of $\sim 1000 \mathrm{H}$ II regions in M33 drawn from the unified catalog of Hodge et al. (2002). These data will eventually yield a high-resolution, two-dimensional metallicity map covering the entire galaxy, with a mean sampling rate of $\gtrsim 2$ measurements per square kpc, and sampling down to $\lesssim 50 \mathrm{pc}$ in some regions. At present, the data set is approximately $20 \%$ of its expected final size.

The observations discussed in this paper were obtained with the LRIS spectrograph (Oke et al. 1995) on the Keck I telescope on 2004 September 22-23 and October 21. Weather conditions during the September nights were clear, while the October observations were affected by clouds at times. The spectrograph was configured with the $600 / 4000$ grism on the blue side, a dichroic at $5600 \AA$, and the $900 / 5500$ and $400 / 8500$ gratings on the red side (in separate exposures). The blue-side spectral resolution was $\sim 5 \AA$, and spectra typically covered $3600-5400 \AA$ with some variation resulting from slit placement on the mask. Slits for which 


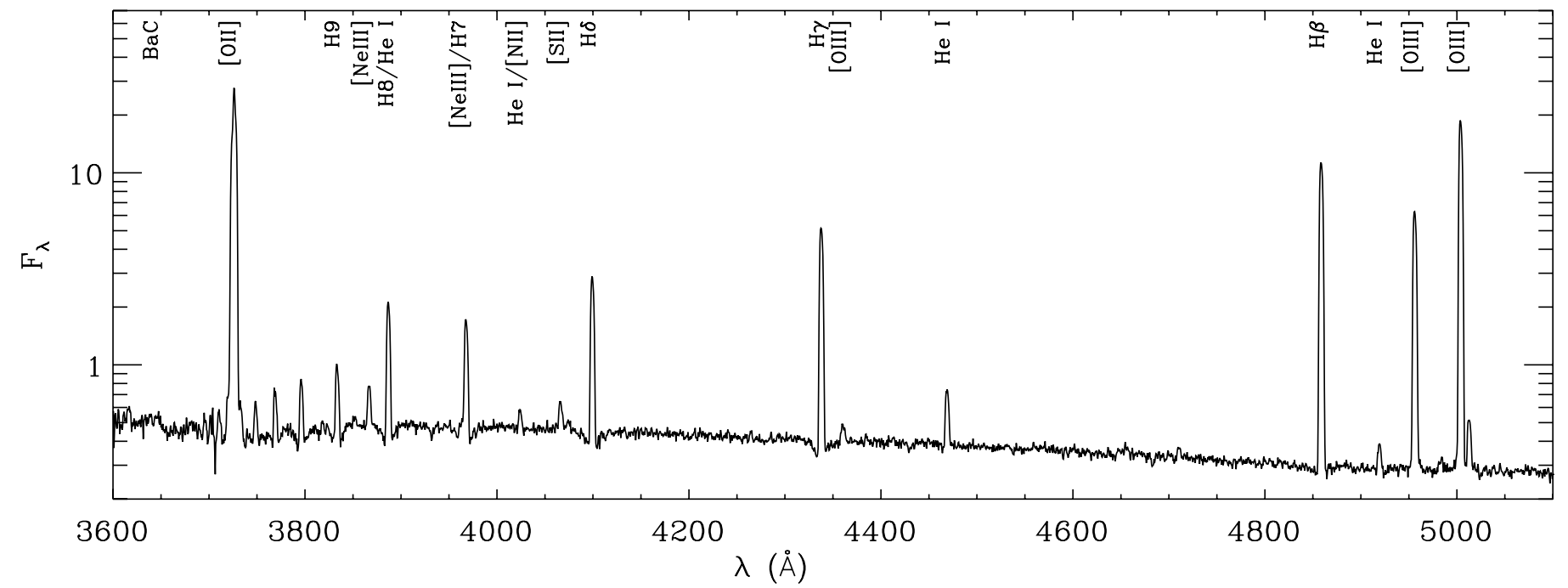

FIG. 1.-Example spectrum of $\mathrm{H}$ II region 60 in Table 1 (BCLMP 266). This spectrum has the median $\mathrm{S} / \mathrm{N}(8.4)$ for the [O III] $\lambda 4363$ line in our sample. Here $F_{\lambda}$ is given in units of $10^{-16} \mathrm{erg} \mathrm{s}^{-1} \mathrm{~cm}^{-2} \AA^{-1}$.

the wavelength coverage missed the [O II] $\lambda 3727$ line have been excluded from our analysis. The slits were $1^{\prime \prime}$ wide and had a minimum length of $10^{\prime \prime}$. We used integration times of 15 minutes per exposure, with 1-4 exposures per slit mask. The slits were generally aligned within $\sim 10^{\circ}$ of the parallactic angle during the observations. We observed 11 slit masks in the southwest half of the galaxy, with an average of $\sim 20$ slits on each mask. For the purposes of this paper we use only the blue-side data, which include all of the lines ([O II] $\lambda \lambda 3726,3729$ and [O III] $\lambda \lambda 4363$, $4959,5007)$ necessary to derive oxygen abundances.

\subsection{Data Reduction}

The data were reduced using an IDL pipeline originally developed for the Sloan Digital Sky Survey spectroscopic data (D. J. Schlegel et al., in preparation) and then modified to deal appropriately with multislit LRIS spectra. The images were biassubtracted and then flat-fielded using either dome flat exposures or the internal quartz lamp. We used the flat-field exposures to identify the location of each slit on the CCD, and the remainder of the processing was carried out on each slit individually. We produced a two-dimensional (2D) wavelength solution for each slit using exposures of $\mathrm{Hg}, \mathrm{Cd}, \mathrm{Zn}, \mathrm{Ne}$, and Ar arc lamps. We then removed cosmic rays from the images and co-added the frames together.

After these basic reduction steps, each slit was examined interactively and the targets were identified based on peaks in the spatial profile of the $\mathrm{H} \beta$ line. We selected an extraction region around each target by following the $\mathrm{H} \beta$ profile down to the background level (which in most cases includes emission from the diffuse ionized medium of M33) and setting the extraction limits where the $\mathrm{H} \beta$ intensity reached the background value on either side of the peak. A B-spline fit to the remainder of the slit produced a 2D sky model covering the entire slit. Finally, we subtracted the sky model from the data and extracted the $\mathrm{H}$ II region spectrum with a boxcar algorithm, yielding a 1D spectrum and associated uncertainties. The spectrum was corrected for atmospheric extinction and flux calibrated by comparison to an exposure of the spectrophotometric standard star G191B2B (Massey et al. 1988). An example calibrated 1D spectrum is shown in Figure 1.

Our procedure for measuring emission-line fluxes in the 1D spectra began by dividing each spectrum up into a series of short
( 200 ̊) spectral windows, each containing at least one bright line (except for the regions between 4500 and $4800 \AA$ and beyond $5100 \AA$ where no such lines exist) and performing a constrained linear + Gaussian fit to all of the emission lines contained within each window. In each window the line separations were fixed at their known values, and the widths of the lines were all set equal to the width of the brightest line in the window (except for blends such as the partially resolved [O II] $\lambda 3727$ doublet). We then measured fluxes by integrating the observed intensity over a $\pm 1.5 \mathrm{FWHM}$ region centered on each line. Uncertainties in the measured line fluxes were calculated by propagating the uncertainty associated with each pixel in the 1D spectrum. We corrected the measured line fluxes for reddening and Balmer absorption from the underlying stellar population using the method outlined by Olive \& Skillman (2001), including all of the Balmer emission lines for which we had high $\mathrm{S} / \mathrm{N}$ detections. We used the Galactic reddening law of Cardelli et al. (1989) and assumed that the equivalent widths of all of the Balmer absorption lines in a given $\mathrm{H}$ II region were the same. We experimented with setting the ratios of the equivalent widths of the absorption lines equal to their mean values averaged over time for the solar metallicity, continuous star formation models of González Delgado et al. (1999), but we found that the resulting metallicity changes are always less than 0.01 dex. With such minimal changes and with possible differences between the stellar populations simulated by González Delgado et al. (1999) and those present in M33 H II regions, we proceeded using the simpler model of constant Balmer equivalent widths. Corrected line fluxes for each $\mathrm{H}$ II region with a detection of [O III] $\lambda 4363$ are given in Table 1 . The listed uncertainties in the line fluxes have been propagated through the reddening and absorption corrections. Balmer line ratios, as well as derived reddening and absorption values, are given in Table 2.

\subsection{Derivation of $\mathrm{H}$ II Region Temperatures and Abundances}

We measure oxygen abundances using the nebular module (Shaw \& Dufour 1995) of the STSDAS package in IRAF. We model the nebula as consisting of two ionization zones with the low- and medium-ionization zones defined by the presence of $\mathrm{O}^{+}$and $\mathrm{O}^{+2}$, respectively. The temperature of the medium ionization zone is calculated using nebular based on the standard ratio of the $[\mathrm{O}$ III $] \lambda \lambda 4959,5007$ doublet to the $[\mathrm{O}$ III] $\lambda 4363$ auroral line. To determine the temperature of the low-ionization zone, 


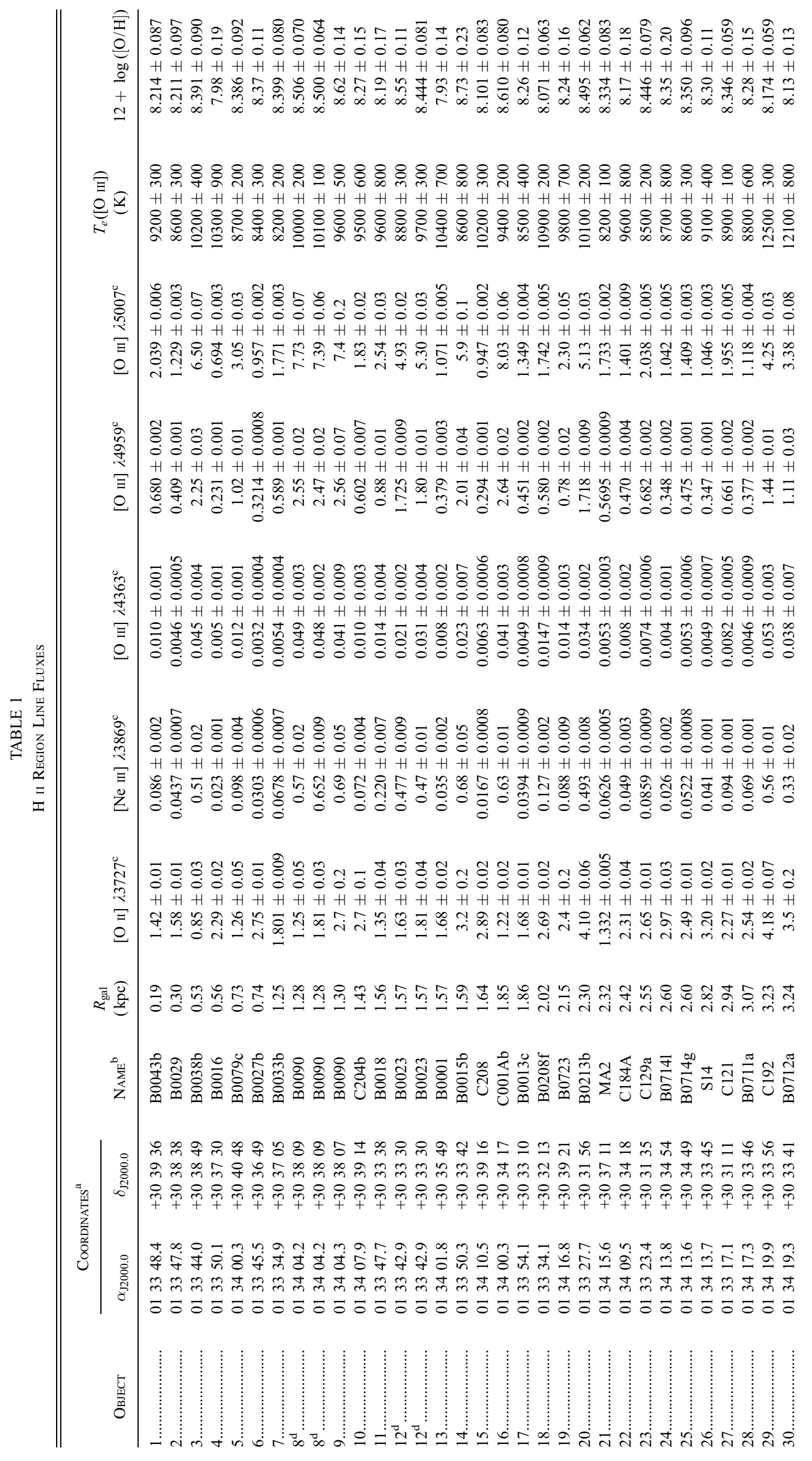


国

어요

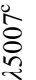

实

○

鼠

当

?ำ

官

อ

oे

官

z

芠

Ð

承总

桿

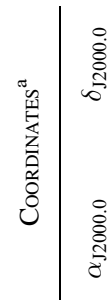

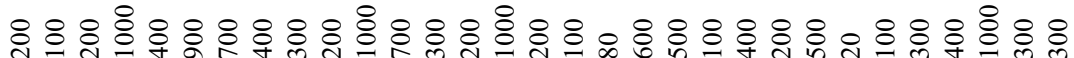

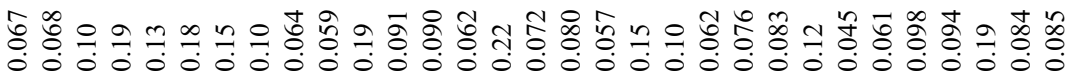

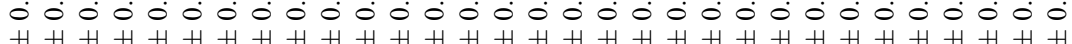
명 o 守

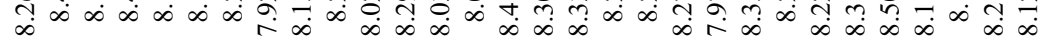

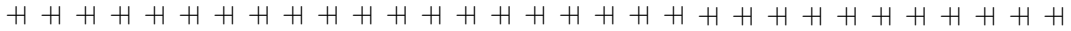

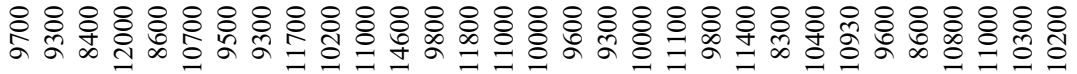

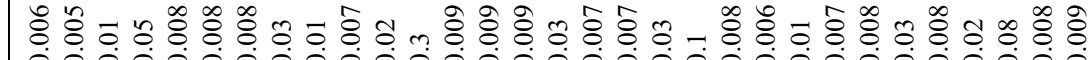

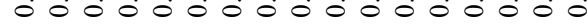
H H H H H H H H H H H H H H H H H H H H H H H H H H H H H H H

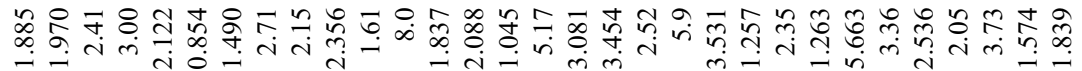

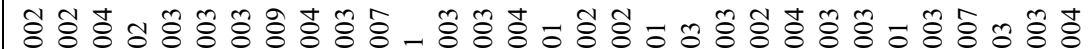
0.00000000000000000000000000000. H H H H H H H H H H H H H H H H H H H H H H H H H H H H H H H 等

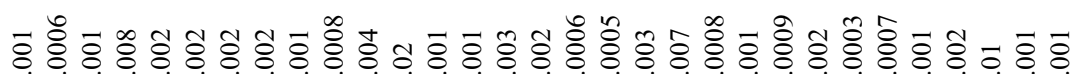
00000000000000000000000000000

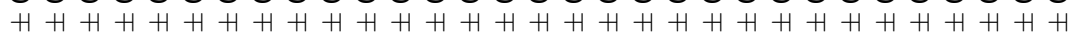

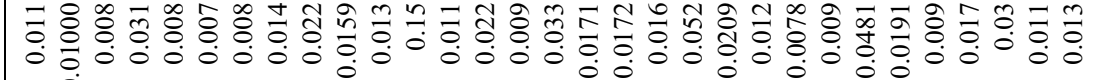

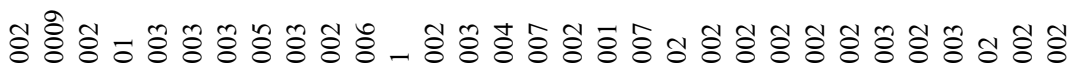
0000000000000000000000000000000 $H+H+H+H+H+H+H+H+H+H+H+H+H$ H H H H H H H H H H H H H

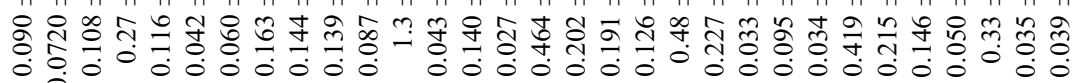

官

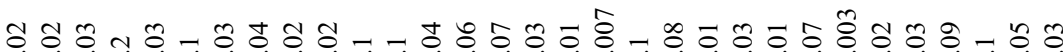
H H H H H H H H H H H H H H H H H H H H H H H H H H H H H H

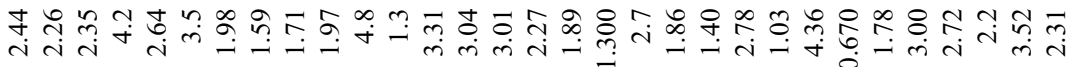

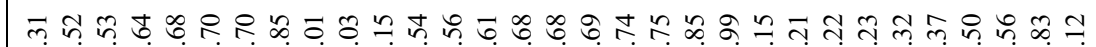

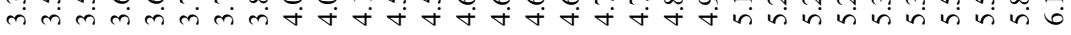

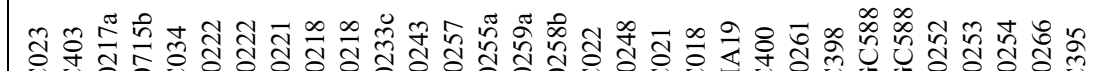

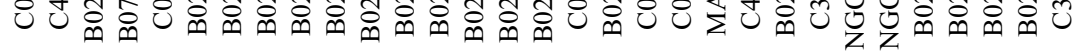

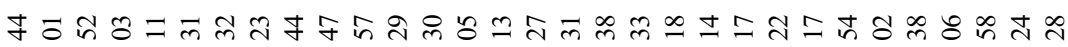
సे

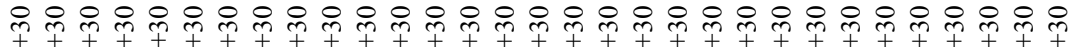

a

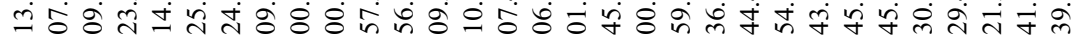
ले

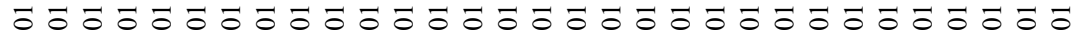

E⿱艹

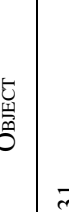

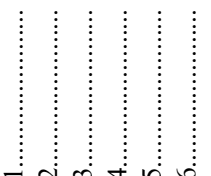<smiles>C1CCCCC1</smiles><smiles>C1CC2CCC2C1</smiles>

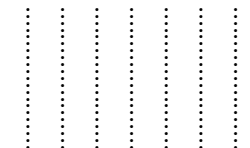

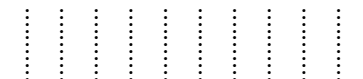

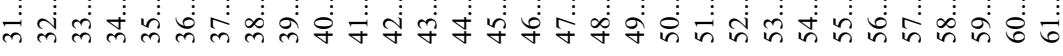


TABLE 2

Balmer Series Line Ratios and Derived Properties

\begin{tabular}{|c|c|c|c|c|c|c|c|c|c|c|c|}
\hline \multirow[b]{2}{*}{ ОвЈест } & \multirow{2}{*}{$\begin{array}{l}\mathrm{EW} \mathrm{H} \beta^{\mathrm{a}} \\
(\AA)\end{array}$} & \multirow[b]{2}{*}{$c(\mathrm{H} \beta)$} & \multirow{2}{*}{$\begin{array}{c}\text { STELlar Absorption EW } \\
(\AA)\end{array}$} & \multicolumn{4}{|c|}{ OBSERVED } & \multicolumn{4}{|c|}{ EXTINCTION/ABSORPTION CORRECTED } \\
\hline & & & & $\mathrm{H} \gamma$ & $\mathrm{H} \delta$ & H7 & H9 & $\mathrm{H} \gamma$ & $\mathrm{H} \delta$ & $\mathrm{H} 7$ & H9 \\
\hline $1 \ldots \ldots \ldots \ldots \ldots \ldots \ldots$ & $135.3 \pm 0.8$ & $0.46 \pm 0.01$ & $2.0 \pm 0.1$ & 0.390 & 0.175 & 0.106 & 0.032 & 0.478 & 0.249 & 0.171 & 0.077 \\
\hline $2 \ldots \ldots \ldots \ldots \ldots \ldots \ldots$ & $268 \pm 2$ & $0.155 \pm 0.008$ & $1.6 \pm 0.1$ & 0.436 & 0.2312 & 0.1200 & 0.0552 & 0.467 & 0.260 & 0.142 & 0.073 \\
\hline $3 \ldots \ldots \ldots \ldots \ldots \ldots \ldots \ldots$ & $310 \pm 30$ & $0.29 \pm 0.04$ & $1 \pm 1$ & 0.416 & 0.213 & 0.239 & 0.044 & 0.47 & 0.26 & 0.30 & 0.07 \\
\hline $4 \ldots \ldots \ldots \ldots \ldots \ldots \ldots . .$. & $190 \pm 3$ & $0.25 \pm 0.01$ & $2.9 \pm 0.2$ & 0.417 & 0.206 & 0.099 & 0.036 & 0.471 & 0.256 & 0.143 & 0.074 \\
\hline $5 \ldots \ldots \ldots \ldots \ldots \ldots$ & $168 \pm 2$ & $0.98 \pm 0.06$ & $7 \pm 1$ & 0.304 & 0.123 & 0.079 & 0.022 & 0.47 & 0.26 & 0.20 & 0.10 \\
\hline $6 \ldots \ldots \ldots \ldots \ldots \ldots$ & $351 \pm 3$ & $0.241 \pm 0.006$ & $1.0 \pm 0.1$ & 0.4303 & 0.2202 & 0.1146 & 0.0566 & 0.473 & 0.255 & 0.139 & 0.074 \\
\hline 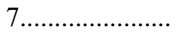 & $357 \pm 3$ & $0.266 \pm 0.006$ & $2.2 \pm 0.2$ & 0.4265 & 0.2112 & 0.1248 & 0.0526 & 0.476 & 0.252 & 0.157 & 0.075 \\
\hline 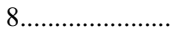 & $640 \pm 10$ & $0.02 \pm 0.05$ & $8 \pm 2$ & 0.441 & 0.229 & 0.301 & 0.044 & 0.47 & 0.26 & 0.34 & 0.07 \\
\hline & $440 \pm 20$ & $0.00 \pm 0.02$ & $2 \pm 2$ & 0.468 & 0.255 & 0.325 & 0.066 & 0.471 & 0.259 & 0.329 & 0.072 \\
\hline $9^{b} \ldots \ldots \ldots \ldots \ldots \ldots$ & $\ldots$ & $0.00 \pm 0.09$ & $11 \pm 2$ & 0.544 & 0.285 & 0.400 & 0.064 & 0.52 & 0.23 & 0.34 & 0.004 \\
\hline $10 \ldots \ldots \ldots \ldots \ldots \ldots$ & $193 \pm 6$ & $0.43 \pm 0.05$ & $2 \pm 1$ & 0.391 & 0.193 & 0.120 & 0.041 & 0.47 & 0.26 & 0.18 & 0.08 \\
\hline $11 \ldots \ldots \ldots \ldots \ldots \ldots \ldots$ & $110.6 \pm 0.5$ & $0.05 \pm 0.03$ & $1.0 \pm 0.7$ & 0.453 & 0.202 & 0.174 & 0.046 & 0.47 & 0.22 & 0.19 & 0.06 \\
\hline $12 \ldots \ldots \ldots \ldots \ldots \ldots$ & $750 \pm 70$ & $0.20 \pm 0.02$ & $2.2 \pm 0.9$ & 0.428 & 0.226 & 0.242 & 0.055 & 0.466 & 0.260 & 0.283 & 0.072 \\
\hline $12 \ldots \ldots \ldots \ldots \ldots$ & $4800 \pm 600$ & $0.15 \pm 0.03$ & $10 \pm 2$ & 0.437 & 0.223 & 0.241 & 0.058 & 0.468 & 0.26 & 0.272 & 0.09 \\
\hline $13 \ldots \ldots \ldots \ldots \ldots \ldots$ & $129.5 \pm 0.3$ & $0.05 \pm 0.01$ & $3.9 \pm 0.2$ & 0.426 & 0.194 & 0.098 & 0.026 & 0.468 & 0.248 & 0.155 & 0.080 \\
\hline $14 \ldots \ldots \ldots \ldots \ldots \ldots$ & $199 \pm 2$ & $1.0 \pm 0.1$ & $3 \pm 2$ & 0.306 & 0.144 & 0.173 & 0.011 & 0.47 & 0.29 & 0.36 & 0.09 \\
\hline $15 \ldots \ldots \ldots \ldots \ldots$ & $203 \pm 1$ & $0.220 \pm 0.008$ & $0.7 \pm 0.1$ & 0.435 & 0.2207 & 0.1261 & 0.0581 & 0.474 & 0.253 & 0.150 & 0.075 \\
\hline $16 \ldots \ldots \ldots \ldots \ldots \ldots$ & $116.0 \pm 0.4$ & $0.00 \pm 0.02$ & $2.5 \pm 0.2$ & 0.456 & 0.222 & 0.283 & 0.042 & 0.479 & 0.254 & 0.316 & 0.083 \\
\hline $17 \ldots \ldots \ldots \ldots \ldots$ & $54.46 \pm 0.08$ & $0.00 \pm 0.01$ & $3.80 \pm 0.04$ & 0.406 & 0.162 & 0.068 & 0.00008 & 0.474 & 0.256 & 0.173 & 0.114 \\
\hline $18 \ldots \ldots \ldots \ldots \ldots \ldots$ & $442 \pm 1$ & $0.09 \pm 0.01$ & $1.6 \pm 0.3$ & 0.452 & 0.238 & 0.156 & 0.063 & 0.471 & 0.256 & 0.172 & 0.074 \\
\hline $19 \ldots \ldots \ldots \ldots \ldots \ldots$ & $240 \pm 10$ & $0.5 \pm 0.1$ & $1 \pm 3$ & 0.382 & 0.142 & 0.082 & 0.014 & 0.47 & 0.20 & 0.13 & 0.03 \\
\hline $20 \ldots \ldots \ldots \ldots \ldots \ldots$ & $248.3 \pm 0.8$ & $0.30 \pm 0.02$ & $0.9 \pm 0.6$ & 0.417 & 0.218 & 0.228 & 0.055 & 0.467 & 0.260 & 0.278 & 0.073 \\
\hline $21 \ldots \ldots \ldots \ldots \ldots \ldots$ & $170.6 \pm 0.3$ & $0.095 \pm 0.004$ & $2.24 \pm 0.04$ & 0.4502 & 0.2154 & 0.1294 & 0.0423 & 0.481 & 0.249 & 0.163 & 0.076 \\
\hline $22 \ldots \ldots \ldots \ldots \ldots$ & $256 \pm 9$ & $0.13 \pm 0.02$ & $0.4 \pm 0.3$ & 0.446 & 0.239 & 0.131 & 0.064 & 0.468 & 0.259 & 0.145 & 0.073 \\
\hline $23 \ldots \ldots \ldots \ldots \ldots \ldots$ & $744 \pm 3$ & $0.270 \pm 0.006$ & $0.0 \pm 0.3$ & 0.426 & 0.2225 & 0.1401 & 0.0622 & 0.470 & 0.257 & 0.165 & 0.075 \\
\hline 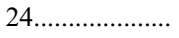 & $350 \pm 10$ & $0.34 \pm 0.01$ & $0.0 \pm 0.2$ & 0.424 & 0.209 & 0.113 & 0.061 & 0.478 & 0.249 & 0.139 & 0.077 \\
\hline $25 \ldots \ldots \ldots \ldots \ldots \ldots$ & $173.4 \pm 0.1$ & $0.288 \pm 0.007$ & $0.80 \pm 0.08$ & 0.4225 & 0.2117 & 0.1258 & 0.0528 & 0.474 & 0.254 & 0.159 & 0.075 \\
\hline $26 \ldots \ldots \ldots \ldots \ldots \ldots$ & $179.7 \pm 0.2$ & $0.14 \pm 0.01$ & $1.9 \pm 0.1$ & 0.436 & 0.219 & 0.1083 & 0.0438 & 0.473 & 0.255 & 0.140 & 0.074 \\
\hline $27 \ldots \ldots \ldots \ldots \ldots \ldots$ & $335 \pm 4$ & $0.150 \pm 0.008$ & $0.7 \pm 0.2$ & 0.446 & 0.2328 & 0.1450 & 0.0631 & 0.473 & 0.255 & 0.162 & 0.074 \\
\hline $28 \ldots \ldots \ldots \ldots \ldots \ldots$ & $435 \pm 8$ & $0.08 \pm 0.01$ & $4.5 \pm 0.4$ & 0.446 & 0.233 & 0.129 & 0.051 & 0.469 & 0.259 & 0.154 & 0.073 \\
\hline $29 \ldots \ldots \ldots \ldots \ldots \ldots . .$. & $125.9 \pm 0.6$ & $0.00 \pm 0.02$ & $3.1 \pm 0.3$ & 0.450 & 0.222 & 0.293 & 0.046 & 0.475 & 0.255 & 0.33 & 0.088 \\
\hline $30 \ldots \ldots \ldots \ldots \ldots \ldots$ & $101 \pm 1$ & $0.08 \pm 0.05$ & $1 \pm 1$ & 0.448 & 0.205 & 0.186 & 0.048 & 0.47 & 0.22 & 0.21 & 0.06 \\
\hline 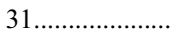 & $191 \pm 3$ & $0.03 \pm 0.01$ & $2.5 \pm 0.2$ & 0.452 & 0.233 & 0.145 & 0.046 & 0.470 & 0.257 & 0.171 & 0.074 \\
\hline $32 \ldots \ldots \ldots \ldots \ldots \ldots$ & $250 \pm 3$ & $0.211 \pm 0.009$ & $2.6 \pm 0.1$ & 0.428 & 0.2123 & 0.1185 & 0.0463 & 0.473 & 0.254 & 0.155 & 0.075 \\
\hline $33 \ldots \ldots \ldots \ldots \ldots$ & $1300 \pm 200$ & $0.31 \pm 0.02$ & $3.6 \pm 0.8$ & 0.420 & 0.208 & 0.130 & 0.052 & 0.475 & 0.253 & 0.167 & 0.075 \\
\hline $34 \ldots \ldots \ldots \ldots \ldots \ldots$ & $820 \pm 70$ & $0.02 \pm 0.06$ & $1 \pm 5$ & 0.462 & 0.222 & 0.180 & 0.073 & 0.47 & 0.23 & 0.19 & 0.08 \\
\hline $35 \ldots \ldots \ldots \ldots \ldots \ldots$ & $237 \pm 1$ & $0.09 \pm 0.02$ & $0.3 \pm 0.3$ & 0.452 & 0.245 & 0.162 & 0.067 & 0.468 & 0.259 & 0.173 & 0.073 \\
\hline $36 \ldots \ldots \ldots \ldots \ldots$ & $146.1 \pm 0.7$ & $0.15 \pm 0.04$ & $2.2 \pm 0.8$ & 0.428 & 0.218 & 0.117 & 0.045 & 0.47 & 0.26 & 0.16 & 0.08 \\
\hline 37..................... & $83.2 \pm 0.2$ & $0.00 \pm 0.02$ & $3.3 \pm 0.2$ & 0.444 & 0.203 & 0.115 & 0.041 & 0.479 & 0.253 & 0.175 & 0.106 \\
\hline $38 \ldots \ldots \ldots \ldots \ldots \ldots \ldots$ & $600 \pm 10$ & $0.90 \pm 0.03$ & $0.9 \pm 4$ & 0.338 & 0.160 & 0.110 & 0.032 & 0.47 & 0.26 & 0.19 & 0.06 \\
\hline 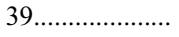 & $162 \pm 3$ & $0.00 \pm 0.02$ & $5.7 \pm 0.2$ & 0.439 & 0.202 & 0.128 & 0.031 & 0.479 & 0.254 & 0.185 & 0.092 \\
\hline $40 \ldots \ldots \ldots \ldots \ldots \ldots$ & $367 \pm 8$ & $0.11 \pm 0.01$ & $4.4 \pm 0.3$ & 0.443 & 0.220 & 0.145 & 0.046 & 0.476 & 0.252 & 0.178 & 0.075 \\
\hline 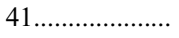 & $280 \pm 30$ & $0.26 \pm 0.04$ & $1 \pm 1$ & 0.421 & 0.205 & 0.115 & 0.025 & 0.47 & 0.24 & 0.14 & 0.04 \\
\hline $42 \ldots \ldots \ldots \ldots \ldots$ & $12.03 \pm 0.07$ & $0.5 \pm 0.1$ & $2.5 \pm 0.2$ & 0.18 & 0.04 & -0.27 & -0.33 & 0.47 & 0.40 & 0.09 & 0.0003 \\
\hline $43 \ldots \ldots \ldots \ldots \ldots \ldots$ & $218 \pm 7$ & $0.10 \pm 0.02$ & $2.0 \pm 0.2$ & 0.448 & 0.228 & 0.140 & 0.052 & 0.474 & 0.254 & 0.166 & 0.075 \\
\hline $44 \ldots \ldots \ldots \ldots \ldots \ldots . .$. & $51.6 \pm 0.2$ & $0.00 \pm 0.03$ & $2.56 \pm 0.06$ & 0.437 & 0.182 & 0.104 & 0.006 & 0.485 & 0.251 & 0.183 & 0.097 \\
\hline $45 \ldots \ldots \ldots \ldots \ldots \ldots$ & $117 \pm 4$ & $0.00 \pm 0.03$ & $4.3 \pm 0.3$ & 0.445 & 0.191 & 0.117 & 0.006 & 0.49 & 0.250 & 0.179 & 0.083 \\
\hline $46 \ldots \ldots \ldots \ldots \ldots \ldots$ & $137 \pm 3$ & $0.00 \pm 0.02$ & $7.0 \pm 0.4$ & 0.425 & 0.193 & 0.244 & 0.037 & 0.469 & 0.259 & 0.301 & 0.109 \\
\hline 47 ........................ & $250 \pm 2$ & $0.054 \pm 0.008$ & $1.6 \pm 0.1$ & 0.456 & 0.2369 & 0.1896 & 0.0558 & 0.473 & 0.255 & 0.209 & 0.074 \\
\hline $48 \ldots \ldots \ldots \ldots \ldots \ldots . .$. & $216.2 \pm 0.2$ & $0.155 \pm 0.007$ & $2.2 \pm 0.1$ & 0.4395 & 0.2167 & 0.1646 & 0.0490 & 0.476 & 0.252 & 0.200 & 0.075 \\
\hline 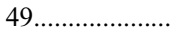 & $119 \pm 4$ & $0.29 \pm 0.06$ & $2.4 \pm 0.8$ & 0.401 & 0.195 & 0.136 & 0.032 & 0.47 & 0.26 & 0.20 & 0.08 \\
\hline $50 \ldots \ldots \ldots \ldots \ldots \ldots$ & $27.6 \pm 0.4$ & $0.25 \pm 0.05$ & $1.5 \pm 0.2$ & 0.373 & 0.124 & 0.000 & -0.045 & 0.47 & 0.23 & 0.10 & 0.06 \\
\hline 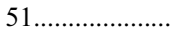 & $214.9 \pm 0.3$ & $0.273 \pm 0.009$ & $3.0 \pm 0.1$ & 0.420 & 0.1923 & 0.1659 & 0.0384 & 0.481 & 0.246 & 0.222 & 0.077 \\
\hline $52 \ldots \ldots \ldots \ldots \ldots \ldots \ldots$ & $138 \pm 2$ & $0.07 \pm 0.02$ & $2.7 \pm 0.2$ & 0.445 & 0.213 & 0.094 & 0.040 & 0.478 & 0.249 & 0.131 & 0.077 \\
\hline $53 \ldots \ldots \ldots \ldots \ldots \ldots$ & $121 \pm 1$ & $0.08 \pm 0.02$ & $6.3 \pm 0.2$ & 0.417 & 0.167 & 0.074 & 0.028 & 0.478 & 0.247 & 0.161 & 0.085 \\
\hline $54 \ldots \ldots \ldots \ldots \ldots \ldots$ & $250 \pm 10$ & $0.18 \pm 0.02$ & $3.2 \pm 0.4$ & 0.432 & 0.211 & 0.101 & 0.044 & 0.475 & 0.252 & 0.136 & 0.076 \\
\hline $55 \ldots \ldots \ldots \ldots \ldots \ldots$ & $455 \pm 3$ & $0.082 \pm 0.005$ & $1.83 \pm 0.07$ & 0.4539 & 0.2316 & 0.2481 & 0.0558 & 0.476 & 0.253 & 0.276 & 0.074 \\
\hline
\end{tabular}


TABLE 2-Continued

\begin{tabular}{|c|c|c|c|c|c|c|c|c|c|c|c|}
\hline \multirow[b]{2}{*}{ ОвЈест } & \multirow{2}{*}{$\begin{array}{c}\mathrm{EW} \mathrm{H} \beta^{\mathrm{a}} \\
(\AA)\end{array}$} & \multirow[b]{2}{*}{$c(\mathrm{H} \beta)$} & \multirow{2}{*}{$\begin{array}{c}\text { Stellar Absorption EW } \\
(\AA)\end{array}$} & \multicolumn{4}{|c|}{ OBSERVED } & \multicolumn{4}{|c|}{ EXTINCTION/ABSORPTION CORRECTED } \\
\hline & & & & $\mathrm{H} \gamma$ & $\mathrm{H} \delta$ & $\mathrm{H} 7$ & H9 & $\mathrm{H} \gamma$ & $\mathrm{H} \delta$ & $\mathrm{H} 7$ & H9 \\
\hline $56 \ldots \ldots \ldots \ldots \ldots \ldots \ldots$ & $3100 \pm 500$ & $0.418 \pm 0.009$ & $10 \pm 10$ & 0.421 & 0.184 & 0.156 & 0.0483 & 0.50 & 0.24 & 0.21 & 0.07 \\
\hline 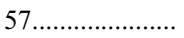 & $243.3 \pm 0.7$ & $0.18 \pm 0.01$ & $0.4 \pm 0.2$ & 0.439 & 0.231 & 0.173 & 0.062 & 0.470 & 0.257 & 0.196 & 0.074 \\
\hline $58 \ldots \ldots \ldots \ldots \ldots \ldots \ldots$ & $117.3 \pm 0.3$ & $0.09 \pm 0.04$ & $5.3 \pm 0.7$ & 0.415 & 0.194 & 0.120 & 0.034 & 0.47 & 0.26 & 0.19 & 0.11 \\
\hline $59 \ldots \ldots \ldots \ldots \ldots \ldots \ldots$ & $20.76 \pm 0.04$ & $0.22 \pm 0.07$ & $1.1 \pm 0.2$ & 0.37 & 0.088 & 0.00 & -0.08 & 0.47 & 0.19 & 0.11 & 0.04 \\
\hline 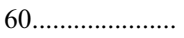 & $164 \pm 4$ & $0.23 \pm 0.02$ & $2.4 \pm 0.2$ & 0.422 & 0.202 & 0.105 & 0.039 & 0.475 & 0.252 & 0.148 & 0.075 \\
\hline 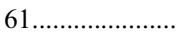 & $95 \pm 1$ & $0.00 \pm 0.01$ & $4.9 \pm 0.1$ & 0.420 & 0.186 & 0.107 & 0.024 & 0.473 & 0.257 & 0.188 & 0.112 \\
\hline
\end{tabular}

Notes. - Table 2 is also available in machine-readable form in the electronic edition of the Astrophysical Journal. Line fluxes are given relative to $\mathrm{H} \beta=1$ and rounded to the precision of one significant figure in the error.

a The equivalent width of $\mathrm{H} \beta$ in emission.

b Because there is no discernible continuum emission associated with the nebular line emission from this $\mathrm{H}$ II region, the equivalent width of the $\mathrm{H} \beta$ emission is undefined.

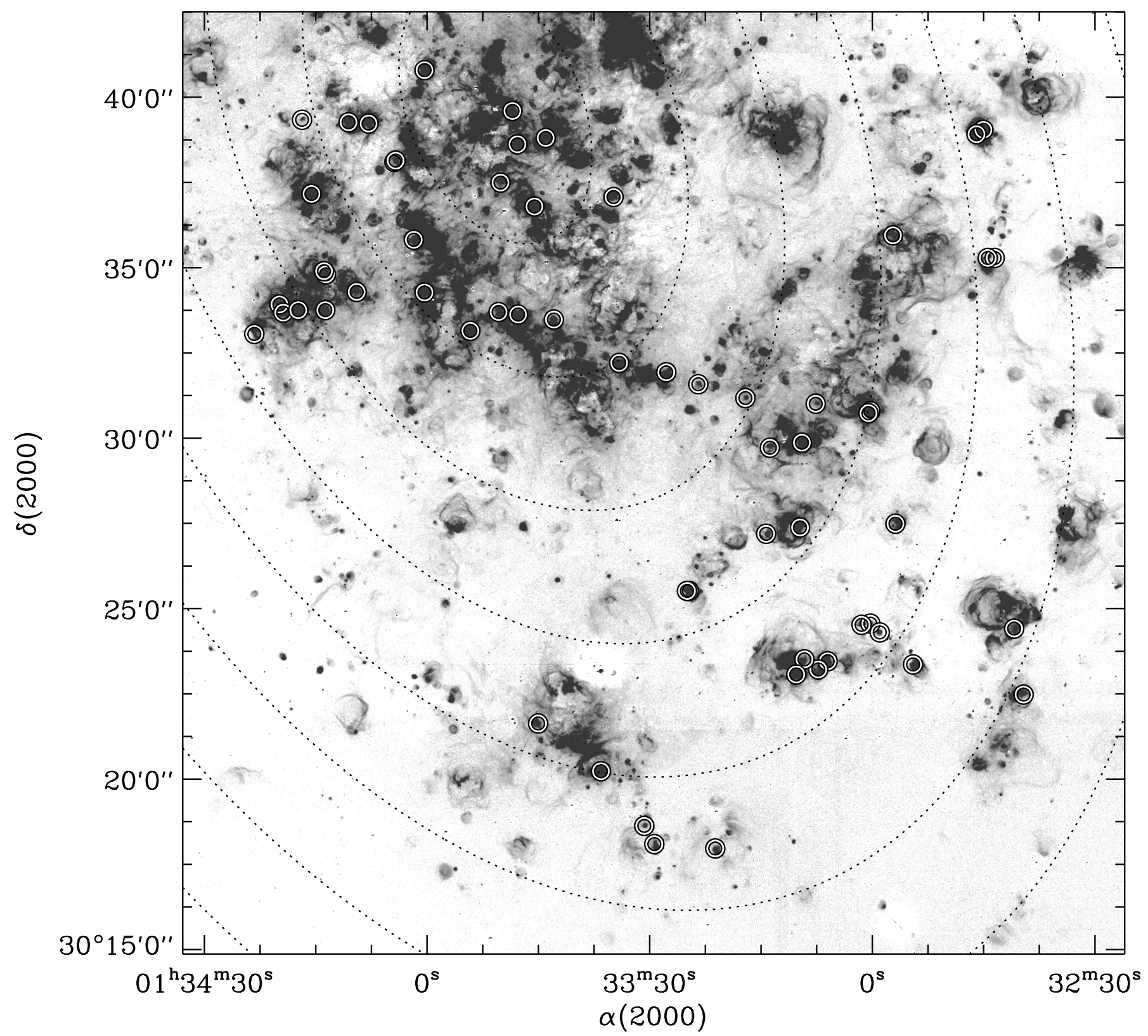

FIG. 2.- Locations of the $61 \mathrm{H}$ II regions with oxygen abundance determinations superimposed on a continuum-subtracted H $\alpha$ image of the southwest por-

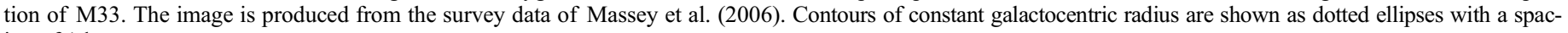
ing of $1 \mathrm{kpc}$. 
TABLE 3

Comparison with Literature Metallicity Measurements

\begin{tabular}{|c|c|c|c|c|c|}
\hline H II Region & KA81 Abundance & V88 Abundance & C06 Abundance & M07 Abundance & Our Abundance \\
\hline NGC 588 & $\ldots$ & $8.30 \pm 0.06$ & $\ldots$ & $\ldots$ & $8.315 \pm 0.061$ \\
\hline MA 2 & 8.38 & $8.44 \pm 0.15$ & $\ldots$ & $\ldots$ & $8.334 \pm 0.083$ \\
\hline 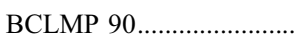 & $\ldots$ & $\ldots$ & $8.50 \pm 0.06$ & $\ldots$ & $8.503 \pm 0.057^{\mathrm{a}}$ \\
\hline 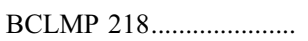 & $\ldots$ & $\ldots$ & $\ldots$ & $8.25 \pm 0.05$ & $8.157 \pm 0.059$ \\
\hline
\end{tabular}

${ }^{\text {a }}$ Weighted average of two independent measurements at the same position; see Table 1 .

References._-(KA81) Kwitter \& Aller 1981; (V88) Vílchez et al. 1988; (C06) Crockett et al. 2006; (M07) Magrini et al. 2007.

we adopt the scaling of Campbell et al. (1986): $T([\mathrm{O} \mathrm{II}])=$ $0.70 T([\mathrm{O}$ III] $])+3000 \mathrm{~K}$. We assume that all $\mathrm{H}$ II regions are in the low density limit $\left(n_{e} \lesssim 10^{2} \mathrm{~cm}^{-3}\right)$. With these densities and temperatures, we measure ionic abundances relative to $\mathrm{H}^{+}$for $\mathrm{O}^{+}$ and $\mathrm{O}^{+2}$ and assume that all oxygen is found in these two ionization states. This analytic approach is common to many extragalactic abundance studies (e.g., Kennicutt et al. 2003), but relies on several (admittedly well justified) assumptions. We will examine these assumptions in future work with the complete sample of $\mathrm{H}$ II regions including the full spectral coverage of LRIS.

We restrict our analysis to the $61 \mathrm{H}$ II regions that have detections of the [O III] $\lambda 4363$ line with $\mathrm{S} / \mathrm{N}>3$ (more conservative selections do not change our results; see $\S 3.1$ ). The locations of these $\mathrm{H}$ II regions are superimposed on an $\mathrm{H} \alpha$ image of the galaxy in Figure 2. All of our targets from this phase of the M33 Metallicity Project are in the southwest portion of the galaxy. Previous work (with the notable exception of Magrini et al. 2007) has focused primarily on giant $\mathrm{H}$ II complexes (e.g., NGC 604, NGC 598) in the northeast half of the galaxy. The [O III] $\lambda 4363$ line is detected in $\sim 1 / 3$ of our targets, and this fraction shows no significant variation with galactocentric radius. Contaminating supernova remnants, emission-line stars, and planetary nebulae are removed from the sample based on identification of characteristic spectral line features and cross-matching with published catalogs of these objects.

As noted in $\S 2.2$, uncertainties in the line fluxes are propagated from single pixel error values. The uncertainties in $c(\mathrm{H} \beta)$ and the equivalent width of the Balmer absorption have been in-

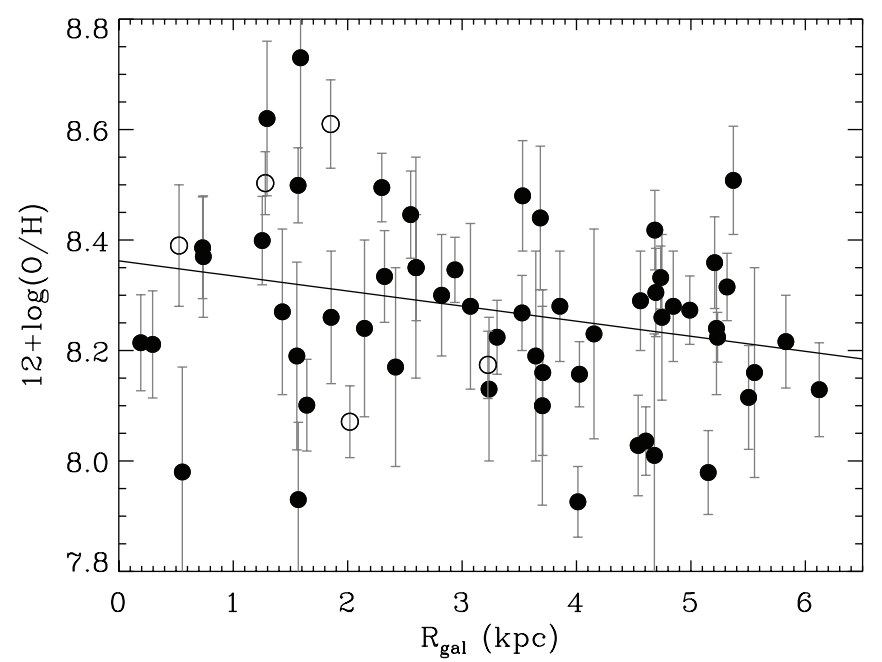

FIG. 3.-Abundances of $61 \mathrm{H}$ II regions in M33 as a function of galactocentric radius. A linear gradient with a slope of $-0.027 \mathrm{dex} \mathrm{kpc}^{-1}$ is fit to the data (solid line). Regions with significant He II $\lambda 4686$ emission are indicated with open symbols. cluded in the errors for the dereddened line fluxes. The reported errors in the physical parameters are determined by a Monte Carlo propagation of errors through the entire metallicity determination process. The Monte Carlo process is particularly well suited for gauging the widths of skewed uncertainty distributions in the derived properties from low signal-to-noise ratio lines. For each $\mathrm{H}$ II region, we generate 100 sets of trial line fluxes by adding a normal deviate times the flux uncertainty to each line flux and reanalyzing the spectrum using nebular. We also include a random fluctuation in the temperature of the low-ionization zone derived from the relationship of Campbell et al. (1986). Based on the magnitude of the uncertainties found in the work of Kennicutt et al. (2003) the $1 \sigma$ error in $T\left(\left[\mathrm{O}_{\mathrm{II}}\right]\right)$ is $300 \mathrm{~K}$. The final uncertainties on the abundances are defined as the $1 \sigma$ widths of the Monte Carlo abundance distributions (see also C06). For reasonably strong [O III] $\lambda 4363$ emission ( $\mathrm{S} / \mathrm{N}>10)$, precisions in the derived metallicity are $\lesssim 0.08 \mathrm{dex}$. For the four $\mathrm{H}$ in regions in our sample that have been studied by previous observers, the abundance determinations agree to within the uncertainties (see Table 3).

\subsection{He II Zones}

We detect He II $\lambda 4686$ from 5 of the $61 \mathrm{H}$ II regions in our sample at a typical level of $2.5 \%$ of the $\mathrm{H} \beta$ flux (objects $3,8,16$, 18, and 29 in Table 1). The emission is almost certainly nebular as it is spectrally unresolved but spatially resolved in these sources (C06). The presence of He II emission indicates that the simple two ionization zone model adopted in the metallicity estimates is somewhat inaccurate. A high-ionization region implies the presence of $\mathrm{O}^{+3}$, which is not accounted for in our abundance determination. Consequently, the derived oxygen abundances will be systematically low by an unknown amount. To estimate the magnitude of this effect, we calculate $\mathrm{He}^{+2} / \mathrm{He}^{+}$ based on the ratio of the $\mathrm{He}$ II $\lambda 4686$ and $\mathrm{He}_{\mathrm{I}} \lambda 4471$ lines. This ratio is only approximate because the $\mathrm{He}$ I line is likely contaminated by stellar absorption. The derived ratio is roughly equal to $\mathrm{O}^{3+} /\left(\mathrm{O}^{+}+\mathrm{O}^{2+}\right)$, providing an estimate of the contribution of $\mathrm{O}^{3+}$. We therefore expect that we have underestimated the oxygen abundance by $\sim 0.01-0.06 \mathrm{dex}$ in these regions. Since we are not sure of the influence of stellar absorption lines, we add (in quadrature) this derived increase to the reported oxygen uncertainty for these five regions.

\section{RESULTS}

\subsection{The Oxygen Gradient}

Figure 3 shows the radial distribution of gas-phase oxygen abundances in M33. We have assumed a thin disk geometry based on a distance to M33 of $840 \mathrm{kpc}$ (Freedman et al. 2001), $i=52^{\circ}$, and P.A. $=22^{\circ}$ (Corbelli \& Salucci 2000). An ordinary, linear least-squares fit to the data, weighting each point according to 


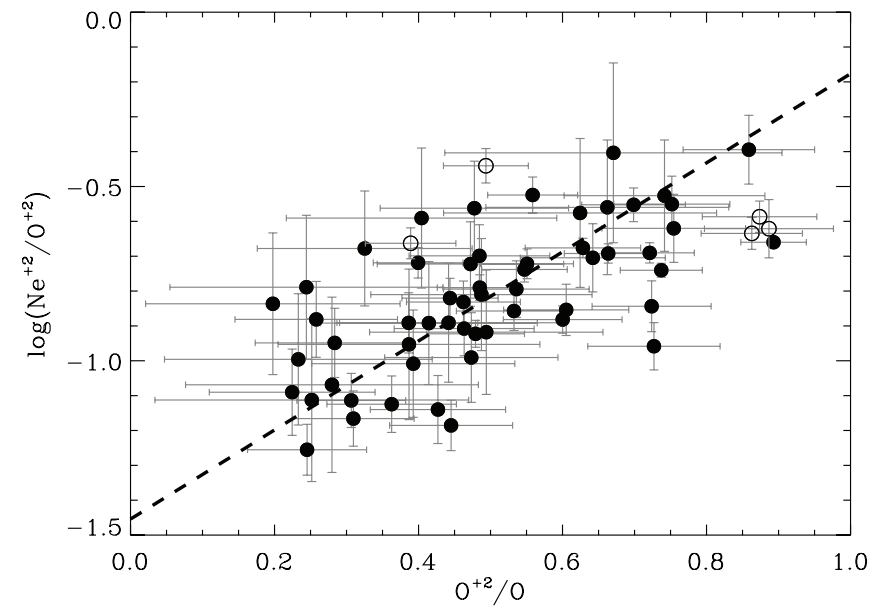

FIG. 4.- Ratio of doubly ionized neon to doubly ionized oxygen plotted as a function of $\mathrm{O}^{+2}$ ionization fraction. Based on photoionization models, this value should be constant and equal to the $\mathrm{Ne} / \mathrm{O}$ ratio. However, there is a clear trend of the ratio between the doubly ionized species and the degree of ionization in real $\mathrm{H}$ II regions. The dashed line shows the fit given in eq. (2). Regions with significant $\mathrm{He}$ II $\lambda 4686$ emission are indicated with open symbols.

its inverse variance, produces a gradient of $12+\log (\mathrm{O} / \mathrm{H})=$ $(8.38 \pm 0.03)-(0.032 \pm 0.006) R_{\mathrm{kpc}}$, but the residuals from this fit are much larger than would be expected from the measurement uncertainties $\left(\tilde{\chi}^{2}=3.2\right)$. Since it is evident that an exponential decline with radius is a reasonable functional form to describe the data, the high $\tilde{\chi}^{2}$ value indicates the presence of substantial intrinsic scatter around the relation.

In this situation, accurately estimating the fit parameters must be done using the method of Akritas \& Bershady (1996, hereafter AB96). We perform a linear regression with the AB96 technique for weighted least-squares fitting in the presence of intrinsic scatter in the data. The AB96 technique estimates the intrinsic variance and adds this variance to that of the data to produce the appropriate weights for the fit. Since the intrinsic variance is larger than the statistical uncertainties for the M33 abundance data, there is less variation in the weights than in the measurement uncertainties. Even though [O III] $\lambda 4363$ detections with $\mathrm{S} / \mathrm{N}$ as low as 3 result in relatively large uncertainties in the derived abundances, including these data improves the quality of the fit by increasing the sample size. This technique yields an abundance gradient of

$$
12+\log (\mathrm{O} / \mathrm{H})=(8.36 \pm 0.04)-(0.027 \pm 0.012) R_{\mathrm{kpc}} .
$$

The implied intrinsic variance in the population (i.e., the scatter in the metallicities at any given radius) is $0.11 \mathrm{dex}$, larger than the precision of most of the measurements. We check the uncertainties on the central abundance and the slope of the abundance gradient by bootstrapping the data (Press et al. 1992), which yields uncertainties $\leqslant 10 \%$ higher than those derived from the AB96 formalism. Although all the included data appear to have a statistically (and visibly) significant [O III] $\lambda 4363$ detection, performing the analysis only on the $23 \mathrm{H}$ il regions with $\mathrm{S} / \mathrm{N}>10$ in this line yields an indistinguishable gradient of $12+\log (\mathrm{O} / \mathrm{H})=$ $(8.41 \pm 0.07)-(0.039 \pm 0.021) R_{\mathrm{kpc}}$. We note that all of our targets are located in southwest half of the galaxy (see Fig. 2), and therefore that the other half of the galaxy could conceivably have a different gradient, but we regard this possibility as unlikely. Finally, we have correlated our derived metallicities and their residuals around a radial gradient against numerous parameters in our analysis such as $L(\mathrm{H} \alpha)$ taken from the catalog of
Hodge et al. (2002), $c(\mathrm{H} \beta)$, the equivalent widths of the Balmer absorption and $\mathrm{H} \beta$ emission, and the oxygen ionization correction factor. We find no correlation with the residuals that would indicate systematic errors in our methods.

\subsection{Complications in Measuring the Neon Gradient}

The slope of the neon abundance gradient is expected to be equal to that of oxygen since these elements are produced in nearly equal proportions for all channels of stellar nucleosynthesis. WNP02 derived neon abundances from Infrared Space Observatory spectra and found a gradient consistent with our oxygen data. Optical observers commonly derive a neon gradient based on the [ $\mathrm{Ne}$ III] $\lambda 3869$ line, yielding an $\mathrm{Ne}^{+2}$ abundance. The fraction of neon found in the $\mathrm{Ne}^{+2}$ state is often assumed to be the same as the fraction of oxygen in $\mathrm{O}^{+2}$, so that an identical ionization correction factor (ICF) can be used for the two elements (Stasińska et al. 2001; C06). Using the simple ICF results, we found a significant gradient in the $\mathrm{Ne} / \mathrm{O}$ ratio, contrary to expectations. We find cause for concern with this assumption for the neon ICF when we derive the abundance of $\mathrm{Ne}^{+2}$ using the standard method and plot the $\mathrm{Ne}^{+2}$ to $\mathrm{O}^{+2}$ ratio as a function of the fraction of oxygen found in the doubly ionized state (Fig. 4). This ratio should be invariant with oxygen ionization fraction and equal to the $\mathrm{Ne} / \mathrm{O}$ ratio, yet the plot shows a significant slope, with $\mathrm{Ne}^{+2} / \mathrm{O}^{+2}$ varying by a factor of $\sim 4$ over the observed range. Since the relationship between the two variables is not known a priori, we fit a linear regression using the BCES method of AB96 to account for the uncertainties in both directions. The resulting fit gives

$$
\log \left(\mathrm{Ne}^{+2} / \mathrm{O}^{+2}\right)=(-1.45 \pm 0.10)+(1.27 \pm 0.22) \mathrm{O}^{+2} / \mathrm{O}
$$

This analysis finds that the slope of the relationship is strongly inconsistent with the value of zero expected from using the same ICF for neon and oxygen. The fraction of doubly ionized oxygen is a proxy for the ionization state of the nebula, so the trend indicates less $\mathrm{Ne}^{+2}$ relative to $\mathrm{O}^{+2}$ in low-ionization nebulae. This is the expected direction for this trend since the third ionization potential of neon $(41 \mathrm{eV})$ is larger than that of oxygen $(35 \mathrm{eV})$. More recent work by Izotov et al. $(2004,2006)$ suggests that applying the oxygen ICF to neon may not be valid in lower ionization regions because of charge transfer reactions between $\mathrm{O}^{2+}$ and atomic hydrogen. These discrepancies could also be caused by a failure to account appropriately for photons with $h \nu \sim 40 \mathrm{eV}$ in the $\mathrm{O}$-star atmospheres used in photoionization codes (C06).

Pérez-Montero et al. (2007) propose a nonlinear ICF based on their own photoionization models:

$$
\mathrm{ICF}\left(\mathrm{Ne}^{2+}\right)=0.753+0.142 x+\frac{0.171}{x},
$$

where $x=\mathrm{O}^{2+} /\left(\mathrm{O}^{+}+\mathrm{O}^{2+}\right)$. Adopting this ICF, we recalculate neon abundances and find no significant gradient in $\mathrm{Ne} / \mathrm{O}$ with radius. For our sample of $\mathrm{H}$ in regions, we measure $\langle\log (\mathrm{Ne} / \mathrm{O})\rangle=$ $-0.69 \pm 0.07$, consistent with the results of Pérez-Montero et al. (2007) for giant extragalactic $\mathrm{H}$ II regions. We conclude that using the same ICF for neon and oxygen is inappropriate for the $\mathrm{H}$ II regions we observed and that more sophisticated ICFs seem to produce reasonable results for generic $\mathrm{H}$ II regions in M33. 


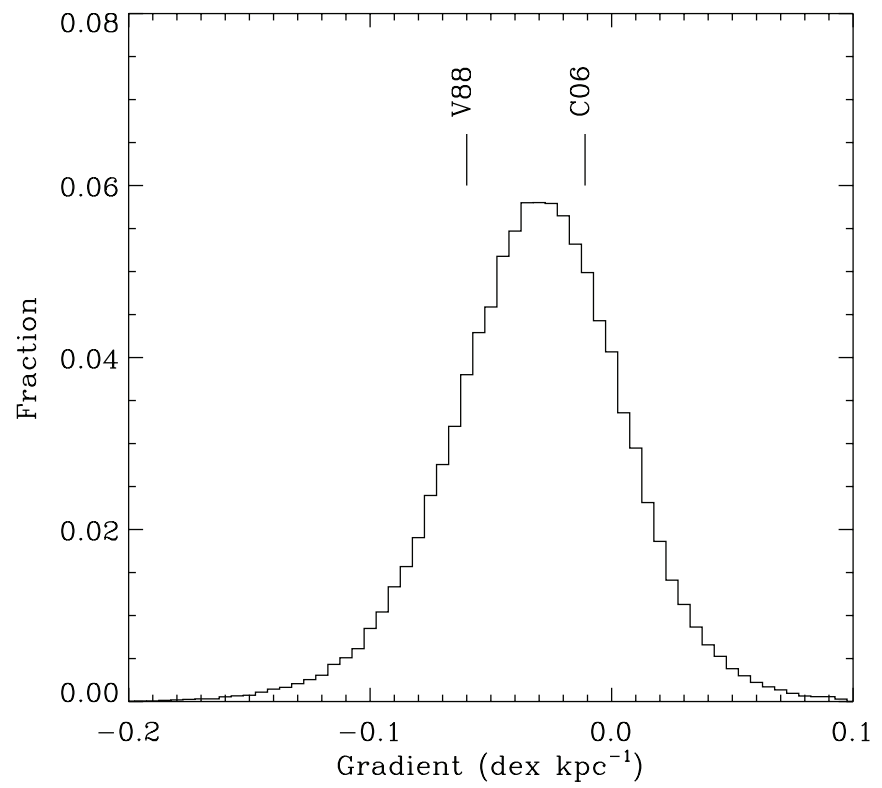

FIG. 5.-Distribution of the abundance gradients that would be measured for samples of $10 \mathrm{H}$ II regions drawn randomly from our sample of 61 . The large width of distribution shows the uncertainty imprinted on the abundance determinations by the underlying variance. The measurements of V88 and C06 are indicated and are consistent with the distribution given their small sample sizes.

\subsection{Comparison to Previous Gradient Measurements}

Our measured gradient of $-0.027 \mathrm{dex} \mathrm{kpc}^{-1}$ is consistent within the uncertainties with the neon gradient of WNP02 $(-0.034 \pm$ $0.015 \mathrm{dex} \mathrm{kpc}^{-1}$ ) and marginally so with the oxygen gradient of $\mathrm{C} 06\left(-0.012 \pm 0.011 \mathrm{dex} \mathrm{kpc}^{-1}\right)$. However, it is not consistent with that of V88 $\left(-0.05 \pm 0.01 \mathrm{dex} \mathrm{kpc}^{-1}\right.$ for the outer regions and -0.10 dex $\mathrm{kpc}^{-1}$ overall after rescaling to our assumed distance) and various older studies. We note that the steep inner gradient measured by V88 is driven by abundances determined from photoionization models, which may be systematically high compared to electron temperature abundances. The apparent discrepancy in abundance gradient likely results, at least in part, from an underestimation of the uncertainties imparted by intrinsic scatter in the $\mathrm{H}$ II region abundances (see $\S 3.4$ ). The intrinsic scatter in our optical $\mathrm{H}$ II region abundances $(0.11 \mathrm{dex})$ is comparable to the value of $0.07-0.10$ dex reported by WNP02.

WNP02 suggested that a linear gradient may not be an appropriate description for the enrichment of M33, arguing for a flat distribution of abundances with a step down at a galactocentric radius of $4 \mathrm{kpc}$. We fit a linear gradient to the $38 \mathrm{H}$ II regions inside $R_{\mathrm{gal}}=4 \mathrm{kpc}$ and find a slope of $-0.015 \pm 0.024 \mathrm{dex} \mathrm{kpc}-1$, which is consistent with no gradient. Stasińska et al. (2006) also claimed an abundance plateau in the inner galaxy based on several subsolar metallicity planetary nebulae in the region. We measure a mean metallicity in the inner galaxy $\left(R_{\text {gal }}<4 \mathrm{kpc}\right)$ of 8.30 ; in the outer galaxy, the mean is 8.21, implying a jump of the same order as, but somewhat smaller than, that of WNP02 if a step function metallicity profile holds.

There now appears to be a growing consensus that the oxygen abundance gradient in $\mathrm{H}$ II regions in M33 is relatively shallow, in conflict with the decades-long assumption of a gradient at least as steep as $-0.10 \mathrm{dex} \mathrm{kpc}^{-1}$. If we ignore the innermost $\mathrm{H}$ II region observed by V88, which does not have a detection of [O III] $\lambda 4363$ or any other temperature-sensitive lines, then all of the modern $\mathrm{H}$ II region studies report gradients of $-0.06 \mathrm{dex} \mathrm{kpc}^{-1}$ or shallower (V88; C06; Magrini et al. 2007). We show in $\S 3.4$ that the results of these studies, which include $6-14 \mathrm{H}$ II region abundances, are consistent with our measurements once their small sample sizes are taken into account.

Other types of sources besides $\mathrm{H}$ II regions are increasingly being employed to study abundances in M33. Urbaneja et al. (2005) used quantitative spectroscopy of OB supergiants to determine an oxygen gradient of $-0.06 \pm 0.02 \mathrm{dex} \mathrm{kpc}^{-1}$. Since these stars are very young, they are expected to trace the interstellar medium (ISM) abundances. If the OB stars have a similar intrinsic abundance scatter to the $\mathrm{H}$ II regions we measure, then this result would be compatible with our findings. Magrini et al. (2007) argue for a steepening of the gradient at small radii based on the young star metallicities in the inner $2 \mathrm{kpc}$ of the galaxy (Urbaneja et al. 2005), but we find no evidence for such a steepening based on the $\mathrm{H}$ II region data alone. Planetary nebulae (Magrini et al. 2004) still yield a steep oxygen gradient $\left(-0.14 \mathrm{dex} \mathrm{kpc}^{-1}\right)$, but this result is dependent on a single nebula at large radius; the remaining objects clearly have a much shallower gradient. Finally, supernova remnants have also shown a shallow oxygen gradient, although the gradients in other elements appear to be steeper (Blair \& Kirshner 1985).

\subsection{Intrinsic Scatter and the Determination of Gradients}

Even after accounting for uncertainties in the stellar absorption and reddening corrections, we find that there is an intrinsic scatter of 0.11 dex around the gradient that is unexplained by the measurement uncertainties. Such scatter is commonly seen in gradient determinations (e.g., in the Milky Way; Afflerbach et al. 1997) and may result from metallicity fluctuations in the interstellar medium. Regardless of its source, gradient determinations made in the face of significant scatter coupled with a limited number of observations may produce widely varying results. The historical evolution of the gradient determination in M33 (one of the best-studied galaxies), ranging over nearly an order of magnitude, serves as a cautionary example.

Only large numbers of measurements can overcome the uncertainties engendered by the intrinsic variance and relatively shallow gradient in M33. In Figure 5 we show the likelihood of measuring a particular value of the gradient if only $10 \mathrm{H}$ II regions from our sample were used in the determination of the gradient. We model the behavior for 10 objects, since this is a typical number of targets studied in estimates of galactic abundance gradients, such as those of V88 and C06. The broad distribution of the derived gradients (standard deviation of $0.03 \mathrm{dex} \mathrm{kpc}^{-1}$ ) suggests that uncertainties in the gradients are systematically underreported in galaxies with significant scatter around their gradients. Even with a sample several times larger than previous studies, we can only report the presence of a gradient in M33 with $2.3 \sigma$ confidence! Other galaxies may have better established gradients owing to smaller intrinsic scatter and/or a steeper abundance gradient (e.g., M101; Kennicutt et al. 2003).

\section{SUMMARY AND CONCLUSIONS}

Using data from the M33 Metallicity Project, we have obtained direct oxygen abundance measurements based on detections of the [O III $\lambda 4363$ line for $61 \mathrm{H}$ II regions in the southwest half of M33. This data set approximately triples the sample of M33 H II region metallicities in the literature and makes M33 by far the best-studied external galaxy in terms of its ISM abundances. We presented a refined oxygen gradient for M33 and found an exponential abundance profile with a gradient of $-0.027 \pm$

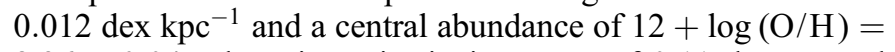
$8.36 \pm 0.04$. There is an intrinsic scatter of $0.11 \mathrm{dex}$ around the trend, which complicates the measurement of the shallow 
gradient in the galaxy. When this scatter is accounted for, previous studies (which generally derived steeper gradients) were shown to be consistent with our measurements. We also noted that there is a significant correlation of the $\mathrm{Ne}^{+2} / \mathrm{O}^{+2}$ ratio with the fraction of oxygen found in the doubly ionized state, which suggests that neon determinations based solely on optical lines require more sophisticated ionization correction factors than are typically assumed.

The authors wish to recognize and acknowledge the very significant cultural role and reverence that the summit of Mauna Kea has always had within the indigenous Hawaiian community. We are most fortunate to have the opportunity to conduct ob- servations from this mountain. We thank the anonymous referee for comments that improved the paper. E. R. acknowledges support from an NSF AAP Fellowship (AST 05-02605), and J. D. S. acknowledges the support of a Millikan Fellowship provided by Caltech. We thank Leo Blitz for support of the early stages of this project, Joe Hennawi for assistance with our data reduction code, and José Vílchez for helpful conversations. We also acknowledge the many useful discussions with the participants in the Metals 2007 Conference whose advice has improved this work. This research has made use of NASA's Astrophysics Data System Bibliographic Services and the SIMBAD database, operated at CDS, Strasbourg, France.

\section{Facilities: Keck:I (LRIS)}

Afflerbach, A., Churchwell, E., \& Werner, M. W. 1997, ApJ, 478, 190

Akritas, M. G., \& Bershady, M. A. 1996, ApJ, 470, 706 (AB96)

Blair, W. P., \& Kirshner, R. P. 1985, ApJ, 289, 582

Boulesteix, J., Courtes, G., Laval, A., Monnet, G., \& Petit, H. 1974, A\&A, 37, 33

Campbell, A., Terlevich, R., \& Melnick, J. 1986, MNRAS, 223, 811

Cardelli, J. A., Clayton, G. C., \& Mathis, J. S. 1989, ApJ, 345, 245

Corbelli, E., \& Salucci, P. 2000, MNRAS, 311, 441

Courtes, G., Petit, H., Petit, M., Sivan, J.-P., \& Dodonov, S. 1987, A\&A, 174, 28

Crockett, N. R., Garnett, D. R., Massey, P., \& Jacoby, G. 2006, ApJ, 637, 741 (C06)

Freedman, W. L., et al. 2001, ApJ, 553, 47

Garnett, D. R., Odewahn, S. C., \& Skillman, E. D. 1992, AJ, 104, 1714

González Delgado, R. M., Leitherer, C., \& Heckman, T. M. 1999, ApJS, 125, 489

Hodge, P. W., Skelton, B. P., \& Ashizawa, J. 2002, An Atlas of Local Group Galaxies (Dordrecht: Kluwer)

Izotov, Y. I., Papaderos, P., Guseva, N. G., Fricke, K. J., \& Thuan, T. X. 2004, A\&A, 421, 539

Izotov, Y. I., Stasińska, G., Meynet, G., Guseva, N. G., \& Thuan, T. X. 2006, A\&A, 448, 955

Kennicutt, R. C., Bresolin, F., \& Garnett, D. R. 2003, ApJ, 591, 801

Kwitter, K. B., \& Aller, L. H. 1981, MNRAS, 195, 939

Lee, M. G., Kim, M., Sarajedini, A., Geisler, D., \& Gieren, W. 2002, ApJ, 565, 959

Magrini, L., Perinotto, M., Mampaso, A., \& Corradi, R. L. M. 2004, A\&A, 426,779

\section{REFERENCES}

Magrini, L., Vilchez, J. M., Mampaso, A., Corradi, R. L. M., \& Leisy, P. 2007, A\&A, 470, 865

Massey, P., Olsen, K. A. G., Hodge, P. W., Strong, S. B., Jacoby, G. H., Schlingman, W., \& Smith, R. C. 2006, AJ, 131, 2478

Massey, P., Strobel, K., Barnes, J. V., \& Anderson, E. 1988, ApJ, 328, 315

Mayall, N. U., \& Aller, L. H. 1942, ApJ, 95, 5

Oke, J. B., et al. 1995, PASP, 107, 375

Olive, K. A., \& Skillman, E. D. 2001, NewA, 6, 119

Pérez-Montero, E., Hägele, G. F., Contini, T., \& Díaz, Á. I. 2007, MNRAS, 381,125

Press, W. H., Teukolsky, S. A., Vetterling, W. T., \& Flannery, B. P. 1992, Numerical Recipes in C: The Art of Scientific Computing (2nd ed.; Cambridge: Cambridge Univ. Press)

Rosolowsky, E., Engargiola, G., Plambeck, R., \& Blitz, L. 2003, ApJ, 599, 258

Searle, L. 1971, ApJ, 168, 327

Shaw, R. A., \& Dufour, R. J. 1995, PASP, 107, 896

Smith, H. E. 1975, ApJ, 199, 591

Stasińska, G., Schaerer, D., \& Leitherer, C. 2001, A\&A, 370, 1

Stasińska, G., Vílchez, J. M., Pérez, E., Gonzalez Delgado, R. M., Corradi, R. L. M., Mampaso, A., \& Magrini, L. 2006, in Planetary Nebulae Beyond the Milky Way, ed. L. Stanghellini, J. R. Walsh, \& N. G. Douglas (Berlin: Springer), 234

Urbaneja, M. A., Herrero, A., Kudritzki, R.-P., Najarro, F., Smartt, S. J., Puls, J., Lennon, D. J., \& Corral, L. J. 2005, ApJ, 635, 311

Vílchez, J. M., Pagel, B. E. J., Díaz, A. I., Terlevich, E., \& Edmunds, M. G. 1988, MNRAS, 235, 633 (V88)

Willner, S. P., \& Nelson-Patel, K. 2002, ApJ, 568, 679 (WNP02) 